\title{
AMHS FACTORS ENABLING SMALL WAFER LOT MANUFACTURING IN SEMICONDUCTOR WAFER FABS
}

\author{
Jesus A. Jimenez \\ Michael Bell \\ Charitha Adikaram \\ Victoria Davila \\ Ingram School of Engineering \\ Texas State University-San Marcos \\ 601 University Drive \\ San Marcos, Texas 78666, USA
}

\author{
Robert Wright \\ Alexander Grosser \\ International SEMATECH Manufacturing Initiative \\ 2706 Montopolis Drive \\ Austin, Texas 78741, USA
}

\begin{abstract}
The automated material handling system (AMHS) may become a critical factor that constrains the capacity of 12-wafer-lot systems. In this paper, AMHS productivity detractors affecting small lot manufacturing are studied, including the track layout, number of vehicles, empty vehicle management rules, number of stockers, stocker capacity, among others. Linked simulation models were developed for the 12-wafer-lot and the 25-wafer-lot systems, using AutoSched AP to simulate wafer processing and AutoMod to simulate the AMHS. The AMHS productivity detractors under study were varied for experimental purposes, and each scenario was quantitatively analyzed. Changes in the factory logistics of the 25 -wafer-lot AMHS led to the development of the 12-wafer-lot AMHS, resulting in less congestion around high-throughput areas and faster AMHS delivery speeds. Simulation results showed improved AMHS performance despite the significant increase in the amount of wafer moves per hour caused by 12 -wafer-lot manufacturing.
\end{abstract}

\section{INTRODUCTION}

The transition of semiconductor wafer fabrication plants (fabs) to small lot manufacturing (SLM) is the next step in the continuous improvement of chip manufacturing. Wafers are currently processed and moved in lots of 25 wafers per carrier, but SLM will use carriers with 12 wafers or less. Several studies have demonstrated that this waste-reduction solution will ultimately improve overall efficiency of production, and thus improve wafer cycle time (Bass and Wright 2008).

In 12-wafer-lot manufacturing, the transport and storage requirements of the Automated Material Handling System (AMHS) will double in relation to the requirements of 25 -wafer-lot manufacturing. Pillai (2006) suggested that high-speed, low variability AMHS will be needed to enable 12-wafer-lot systems. However, the AMHS is currently a productivity detractor of small lot size operations because existing transport technologies provide delivery speeds that are too low to maintain the increased move requirements. The AMHS also has high dependence on centralized storage systems, which leads to unnecessary congestion and increases the AMHS inefficiencies.

With this in mind, the purpose of this paper is to configure the AMHS factors that limit the capacity of 12-wafer-lot systems, seeking to optimize the fab logistics and minimize traffic congestion around high-throughput areas of the fab. To accomplish this purpose, linked simulation models of a virtual $300 \mathrm{~mm}$ fab were developed in AutoSched AP and AutoMod. These models study at a higher level of de- 
tail the interactions that occur between the wafer processing equipment and the AMHS, thus providing more accurate estimations of the fab performance metrics.

The remaining part of this paper is as follows. Section 2 presents the related literature review. Section 3 describes the characteristics of the $300 \mathrm{~mm}$ virtual fab under study. Section 4 provides an analysis of the AMHS productivity detractors in 25-wafer-lot wafer fabs. Similarly, Section 5 provides an analysis and configuration of the AMHS productivity detractors to enable 12-wafer-lot manufacturing. Section 6 summarizes the conclusions of this study and explains our future work.

\section{LITERATURE REVIEW}

The published work available in the literature attempts to study the feasibility of small lot manufacturing in semiconductor wafer fabs. Marshall, Rust, and Schmidt (2008) identified a number of productivity detractors that need to be addressed for the next generation of factories. The productivity detractors included batching and cascading methodology, setup time and first-wafer delays, AMHS delivery and tool buffering, etc. The authors proposed 12-wafer lots as a waste reduction solution. Pettinato and Pillai (2005) performed a preliminary analysis of factors that will contribute to the conversion of factories from $300 \mathrm{~mm}$ wafers to $450 \mathrm{~mm}$, and suggested that $450 \mathrm{~mm}$ wafer fabs will integrate SLM operations. Adusumilli and Wright (2004) conducted a simulation study on standard Front Opening Unified Pod (FOUP) capacities of 25 and 13. Results showed that the use of the 13-wafer FOUP did not provide conclusive evidence that the lower lot size was favorable over a larger lot size. Wright and Chang (2006) discussed the advantages and disadvantages of small transfer batch sizes in a semiconductor factory. They establish a method to determine the theoretical cycle time from the prospective of the wafer. More commonly the cycle time is determined from the perspective of the lot. Their study provided an opportunity for reduction of cycle time by minimizing transfer batch sizes.

There is a lack of papers that study the impact of the AMHS in SLM fab operations. Pillai (2006) indicated that the next generation transport systems for the small lot manufacturing model must have higher transport capabilities than existing vehicle-based solutions. Glüer (2003) discussed the importance of optimizing AMHS operations due to the high amount of move transaction observed in megafabs (i.e. fabs with throughput levels of 100,000 wafer starts per month or more). A MaxFlow algorithm is presented as a preliminary step for designing the AMHS layout. Zimmerhackl et al. (2007) investigated the effects of small lot manufacturing on factory performance. Their analysis showed an increase of up to $23 \%$ in the reduction of cycle time. Authors identified the need of further studies on AMHS operation, equipment front-end design, and FOUP handling scenarios.

\section{DESCRIPTION OF SYSTEM AND CORRESPONDING SIMULATION MODELS}

Texas State University-San Marcos and International SEMATECH Manufacturing Initiative (ISMI) combined modeling initiatives to assess and ensure $300 \mathrm{~mm}$ production of a virtual manufacturing fab. Linked Capacity/AMHS models were developed in AutoSched AP (version 9) and AutoMod (version 12) to simulate the 12-wafer-lot and 25-wafer-lot systems. These models were concepts driven by SEMATECH'S Factory for Small Lot Size (FSLS) project in 2008/2009.

The AutoSched AP model infrastructure is made up of 3 different technologies, including $32 \mathrm{~nm}$, $45 \mathrm{~nm}$, and $65 \mathrm{~nm}$. The least advanced technology, $65 \mathrm{~nm}$, is represented by 3 process flows with a total of 8,9 , and 10 metal levels, each of which is modeled as an individual process route in wafer manufacturing model. The release rate is 30,000 wafer starts per month (wspm) for product wafers and 4,500 wspm for non-product wafers or test-wafers. Each processing route, 5 total, consists of approximately 500 to 700 processing steps. In addition, there are 54 different workstations and about 700 tools.

The AutoMod models have the following characteristics:

- Bays are arranged along a central spine layout with an inner loop and two outer loops. The layout dimensions are shown in Figure 1. 
- The fab has a 38-bay configuration. Each bay can hold up to 24 tools. Figure 2 provides a graphical representation of the tool distribution organized by bay and tool position.

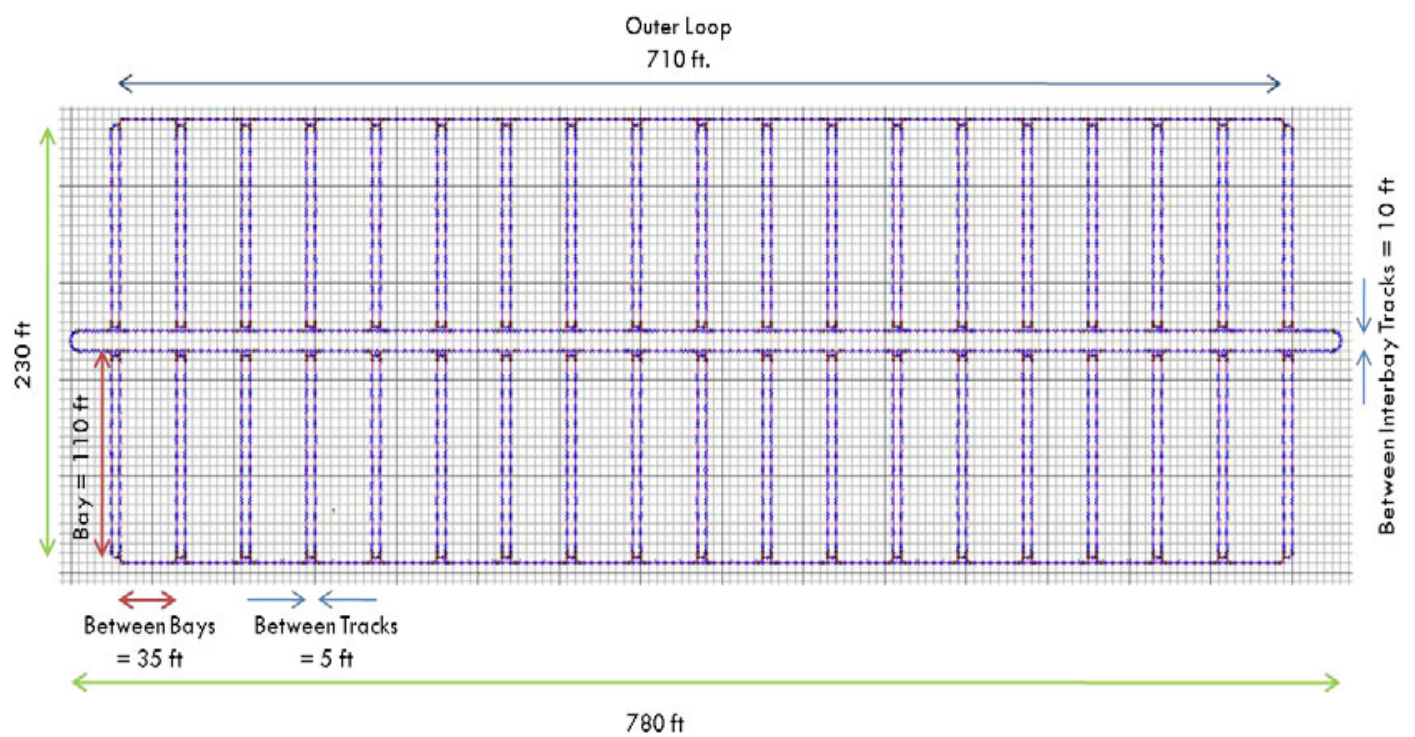

Figure 1: Spine layout configuration for the $300 \mathrm{~mm}$ virtual fab

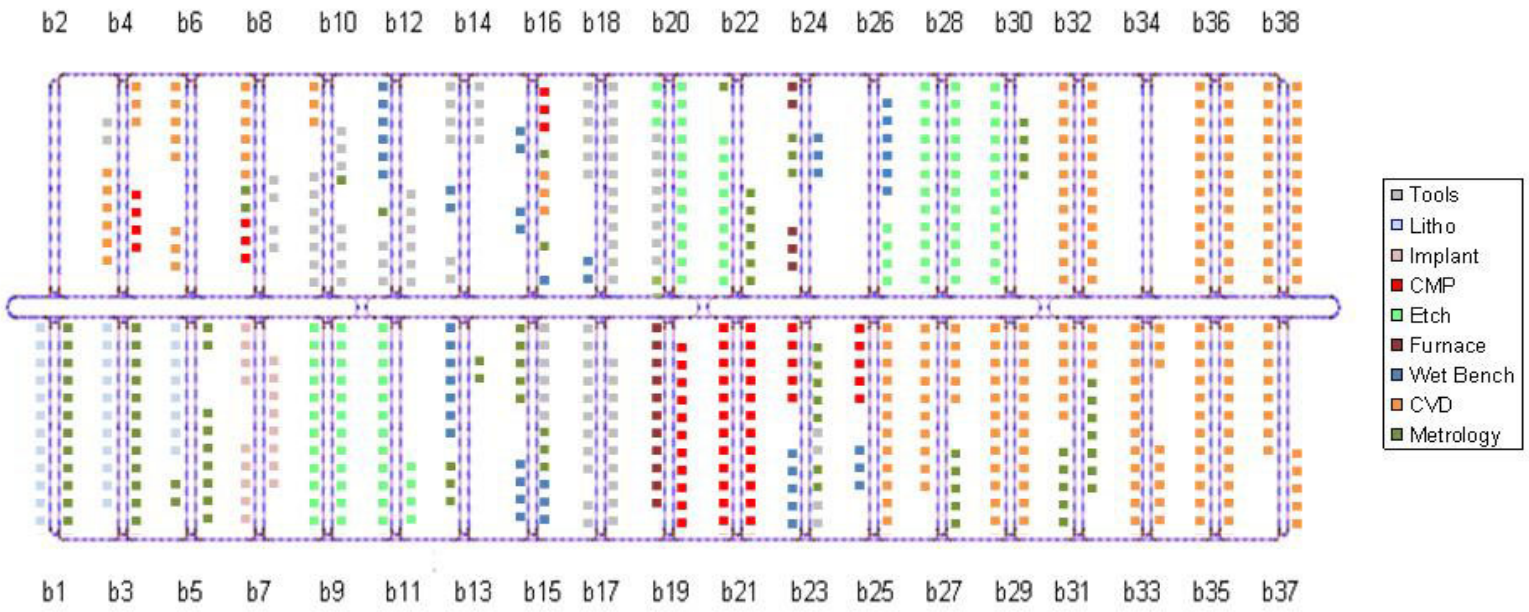

Figure 2: Tool type organized by location

- Tools have finite tool port capacity.

- The AMHS performs tool-to-tool (TtT) moves and tool-to-stocker/stocker-to-tool (TtS/StT) moves.

- Vehicles travel at a forward velocity of $6.6 \mathrm{ft} / \mathrm{sec}$ and at a curve velocity of $2.4 \mathrm{ft} / \mathrm{sec}$.

- AMHS tracks are unidirectional.

- Vehicle hoist time is $7.5 \mathrm{sec}$. for a complete cycle, which includes extending and retracting the vehicle's hoist to load/unload a carrier.

- The number of vehicles ranges between 125-250 vehicles.

- The number of stockers ranges between 12-24 stockers.

- Each stocker has a finite storage capacity.

- Each stocker has one input and one output port. 
Jimenez, Wright, Grosser, Bell, Adikaram, and Davila

\section{ANALYSIS OF 25-WAFER-LOT SYSTEMS}

\subsection{Experimental Design}

Critical AMHS factors constraining wafer processing were identified in this study. These factors are summarized in Table 1. A series of linked models (i.e. Models I-VII) were developed by changing the corresponding factor levels.

Table 1: Summary of experimental factors and factor levels used within the 25-wafer-lot linked model

\begin{tabular}{|c|c|c|c|c|c|c|c|c|c|}
\hline \multirow[b]{2}{*}{ Code } & \multirow[b]{2}{*}{ Factor } & \multirow{2}{*}{$\begin{array}{c}\text { ASAP } \\
\text { Model } \\
\text { Baseline }\end{array}$} & \multicolumn{7}{|c|}{ Linked Wafer Processing/AMHS Model } \\
\hline & & & $\mathbf{I}$ & II & III & IV & $\mathbf{V}$ & VI & VII \\
\hline $\mathrm{A}$ & \# of Vehicles & $\infty$ & 200 & 200 & 200 & 150 & 125 & 150 & 125 \\
\hline $\mathrm{B}$ & \# of Stockers & 30 & 12 & 12 & 12 & 12 & 12 & 12 & 12 \\
\hline $\mathrm{C}$ & Stocker capacity & $\infty$ & 9600 & 9600 & 2400 & 2400 & 9600 & 2400 & 2400 \\
\hline $\mathrm{D}$ & Tool-to-tool move rate & $0 \%$ & $47 \%$ & $27 \%$ & $25.4 \%$ & $25.4 \%$ & $47 \%$ & $25.2 \%$ & $25.2 \%$ \\
\hline $\mathrm{E}$ & Inner loop & None & Single & Single & Single & Single & Single & Single & Single \\
\hline $\mathrm{F}$ & Outer loop & None & Single & Single & Single & Single & Single & Single & Single \\
\hline $\mathrm{G}$ & Empty vehicle control & None & $\mathrm{VDB}^{1}$ & $\mathrm{VDB}^{1}$ & $\mathrm{VDB}^{1}$ & $\mathrm{VDB}^{1}$ & $\mathrm{VDB}^{1}$ & $\mathrm{ZB}^{2}$ & $\mathrm{ZB}^{2}$ \\
\hline
\end{tabular}

1. $\mathrm{VDB}=$ Vehicle Dedication to Bay 2. $\mathrm{ZB}=$ Zone Balancing

\subsection{Summary of Results}

The 25-wafer-linked models (Models I-VII) were simulated for 750 days, which includes a warm-up period of 250 days. Several snaps were collected during the simulation run. Each model was stable. The performance statistics that were analyzed included delivery time (broken down into retrieve and transport times), moves per hour, stocker load, and vehicle utilization, among others. The results of these simulations are summarized in Table 2, organized by model (rows) and performance statistics (columns).

Table 2: Summary of simulation results for the 25-wafer-lot linked model

\begin{tabular}{|c|c|c|c|c|c|c|}
\hline Model & $\begin{array}{c}\text { Vehicle } \\
\text { Utilization } \\
(\%)\end{array}$ & $\begin{array}{c}\text { Avg. Stocker } \\
\text { Load } \\
\text { (lots) }\end{array}$ & $\begin{array}{c}\text { Total Stocker } \\
\text { Load } \\
\text { (lots) }\end{array}$ & $\begin{array}{c}\text { Moves } \\
\text { Per } \\
\text { Hour }\end{array}$ & $\begin{array}{c}\text { Retrieval } \\
\text { Time } \\
\text { (sec.) }\end{array}$ & $\begin{array}{c}\text { Transport } \\
\text { Time } \\
\text { (sec.) }\end{array}$ \\
\hline $\begin{array}{c}\text { I: }(200 \mathrm{veh} / 12 \mathrm{stk} / \\
9600 \mathrm{stk} \mathrm{cap} / \mathrm{VDB})\end{array}$ & 21 & 57 & 684 & 1393 & 60 & 84 \\
\hline $\begin{array}{c}\text { II: }(200 \mathrm{veh} / 12 \mathrm{stk} / \\
9600 \mathrm{stk} \mathrm{cap} / \mathrm{VDB})\end{array}$ & 26 & 58 & 705 & 1716 & 100 & 91 \\
\hline $\begin{array}{c}\text { III: }(200 \mathrm{veh} / 12 \mathrm{stk} / \\
2400 \mathrm{stk} \mathrm{cap} / \mathrm{VDB})\end{array}$ & 25 & 51 & 614 & 1715 & 73 & 87 \\
\hline $\begin{array}{c}\text { IV: }(150 \mathrm{veh} / 12 \mathrm{stk} / \\
2400 \mathrm{stk} \mathrm{cap} / \mathrm{VDB})\end{array}$ & 36 & 57 & 689 & 1713 & 102 & 85 \\
\hline $\begin{array}{c}\text { V: }(125 \mathrm{veh} / 12 \mathrm{stk} / \\
9600 \mathrm{stk} \mathrm{cap} / \mathrm{VDB})\end{array}$ & 33 & 59 & 707 & 1394 & 95 & 82 \\
\hline $\begin{array}{c}\text { VI*: }(150 \mathrm{veh} / 12 \mathrm{stk} / \\
2400 \mathrm{stk} \mathrm{cap} / \mathrm{ZB})\end{array}$ & 56 & 53 & 636 & 1723 & 133 & 86 \\
\hline $\begin{array}{c}\text { VII*: }(125 \mathrm{veh} / 12 \mathrm{stk} / \\
2400 \mathrm{stk} \mathrm{cap} / \mathrm{ZB})\end{array}$ & 69 & 52 & 622 & 1717 & 136 & 83 \\
\hline
\end{tabular}


According to Table 2, Cycle Time ranges between 46.3 and 47.99 days. The final 25-wafer-lot models were simulated with both 125 and 150 vehicles, using the ZB rule, which are intended to be representative of the utilization in today's factories.

\subsubsection{Vehicles}

Prescreening experiments with the linked model demonstrated that the number of vehicles should be between 125 and 250 vehicles for a given configuration to produce feasible and reasonable fab performance. According to Table 2, the number of vehicles affects the amount of time to respond to a move request (i.e. retrieve time) and the time to transfer a carrier between two processing steps (i.e. transport time). In general, a small supply of vehicles increase retrieve time, but it also decreases transport time. However, a large supply of vehicles decreases retrieves time, but it also increases transport time. Higher retrieve times tend to accumulate more carriers at stockers. These observations are made by analyzing three different comparisons (i.e., Model I vs. Model V, Model III vs. Model IV, and Model VI vs. Model VII).

\subsubsection{Stockers}

The number of stockers is a factor that also affects the retrieve time and transport time. In a system with fewer stockers, most stockers process a larger amount of carriers. As a result, carriers experience longer delays at the stocker waiting to access the stocker's output port. A large amount of vehicles accumulate around the stocker, waiting to pick up and drop off carriers. Furthermore, with fewer stockers, carriers will travel longer distances if the stocker is not strategically located near destination tools. Increasing the number of stockers, on the other hand, should reduce these time delays, and thus decrease the overall delivery time. The 25-wafer-lot linked model has 12 stockers.

\subsubsection{Stocker capacity}

Each of the 12 stockers represented in the system was constrained initially at 800 lots, providing a total storage capacity of 9600 lots. Preliminary simulation results indicate that there is approximately $317 \%$ more storage capacity in this scenario than the average WIP level (which is about 2300 lots for the 25wafer-lot linked model).

A more conservative scenario was designed by reducing the total stocker capacity to 2400 lots. Such total storage capacity represents only about $4 \%$ more that the average WIP level. In this linked model, tools were dedicated to a primary stocker. Individual stocker capacities fluctuate between 100 and 300 lots. Each stocker capacity was determined based on the number of tools dedicated to the stocker, as well as the tool's move settings. Several iterations of simulations were required prior to determining the stocker's optimal capacity levels, particularly because some stockers reached their maximum storage capacity during the simulation. In other words, the stocker was dedicated to more tools than the capacity of the stocker.

In general, reducing the stocker capacity from 9600 to 2400 lots leads to lower retrieve and transport times. This effect is expected because there are fewer carriers per stocker load port constraining other carriers to access a vehicle. The transport time is lower too because there is less congestion around stockers, thus decreasing the utilization of vehicles. These conclusions are made by comparing Model II vs. Model III.

\subsubsection{Tool-to-tool move rate}

As was discussed above, another way to over utilize stockers is by changing the move settings (i.e. toolto-tool vs. tool-to-stocker/stocker-to-tool settings). A tool configured with a tool-to-tool setting receives lots directly from an upstream tool. However, if there is no capacity at the tool load ports, carriers will go to the stocker assigned to the tool family. Alternatively, for a tool configured with a tool-to- 
stocker/stocker-to-tool setting, carriers must go to the stocker that is assigned to the tool before it travels to the destination tool. In either case, the destination of the carrier will depend on the downstream tool's policy after the current processing step is completed. This study considers tool-to-tool move rates of approximately $25 \%$ and $45 \%$.

Increasing the rate of tool-to-tool deliveries results in less moves per hour required to be processed by the AMHS. The reader should note that the total number of moves per hour metric represents the sum of individual tool-to-tool, tool-to-stocker, and stocker-to-tool transfers. Our simulation results show that retrieval time, transport time, and vehicle utilization drops because there is less congestion in the system. These conclusions are made by comparing Model I vs. Model II.

\subsubsection{Inner loop}

The inner loop is unidirectional, following a counterclockwise direction. The inner loop unites the bays around the center of the fab, enabling vehicles to access a bay from all other bays. There are two linear tracks in the inner loop, each with a length of $770.0 \mathrm{ft}$. In addition, there are two curvilinear sections uniting the linear sections at the two extremes (each with a length of $15.7 \mathrm{ft}$.); and six crossovers (each with length of $15.7 \mathrm{ft}$.). The total length of the inner loop is $1665.7 \mathrm{ft}$.

\subsubsection{Outer loop}

The outer loop is also unidirectional, following a clockwise direction. The outer loop unites the bays from the outside. Vehicles can only access certain bays by using the outer loop. There are two linear tracks in the outer loop, each with a length of $710.0 \mathrm{ft}$.

\subsubsection{Empty vehicle control rules}

Empty vehicle management is critical for an effective AMHS performance. After completing the delivery of a carrier, an AMHS vehicle needs to find a location where to wait until it is claimed by other carriers. An empty vehicle should therefore be strategically positioned in the fab by minimizing the travel time (or distance) to the next assigned "pick-up" job. Managing empty vehicles also contributes to less vehicle congestion, higher vehicle utilization, and lower waiting times for vehicles. In this project, we studied two empty vehicle control rules: Vehicle Dedicated to Bays (VDB) and Zone Balancing (ZB).

\subsubsection{Vehicle Dedicated to Bays}

This management policy models a pessimistic case in which vehicle resources are ineffectively consumed. Under the VDB rule, empty vehicles are assigned to the interbay or one of the 38 bay loops. Vehicles operating in a bay loop are capable of performing tool-to-tool and tool-to-stocker deliveries, whereas the vehicles operating in the interbay loop are capable of performing stocker-to-tool deliveries only. Empty vehicles travel around the assigned loop, also referred to as the home loop, until a carrier claims it.

Once a move request is received, the empty vehicle picks up the carrier at the tool or stocker. The vehicle loads the carrier, and transfers it to the destination location. Then, the vehicle returns "empty" to its home loop after completing its delivery task, where it checks for any unassigned carriers.

\subsubsection{Zone Balancing (ZB)}

The idea behind ZB is removing unnecessary delays caused by dedicating vehicles to bays (i.e. congestion caused by returning empty vehicles to home bay). In the ZB rule, the main loop and the bays are denominated zones. There are therefore 39 zones in the fab. Empty vehicles do not return to home, as was observed in the VDB rule, but instead they are allowed to remain at the zone where they complete a deli- 
very job. Alternatively, these vehicles may be sent to other zones to pick up high-priority jobs (carriers that have not been able to claim an empty vehicle due to an absence of vehicles at the zone).

To avoid an undersupply or oversupply of empty vehicles, the zone's number of vehicles is constantly compared against certain predefined levels, called watermarks. There is a high watermark level and low watermark level defined for each zone. The function of the low watermark level is to pull empty vehicles from other zones if the number of empty vehicles at the zone falls below this level. This effect occurs when the rate at which vehicles leave the zone is higher than the rate at which empty vehicles become available at the zone (e.g. after completing a delivery at the current zone). Maintaining a solid base of empty vehicles at the zone, such as that enabled by an adequate low-watermark level, is a critical contributor to reducing the time that carriers wait for empty vehicles.

On the other hand, an oversupply of empty vehicles at the zone leads to congestion and low vehicle utilizations. The function of the high water mark level is to avoid accumulating empty vehicles at the bay. When the number of empty vehicles exceeds the high watermark level, then those vehicles in excess are sent to the main loop system. This effect occurs when the rate at which vehicles empty vehicles become available at a zone is higher than the vehicles that depart the zone.

In this study, vehicles have higher utilizations operating under the ZB-based management system than those vehicles operating under the VDB-based management system. The comparison is based on Models IV and VI.

\subsection{Lessons learned on 25-wafer-lot models}

The modeling of the 25-wafer modeling was challenging. Significant model development was done in order to achieve stable models. The top critical changes to the 25 -wafer-linked models as the simulations progressed were:

1 Optimizing the rate of tool-to-tool moves, as well as determining the tools to be configured with a tool-to-tool setting.

2 Providing sufficient track length for the AMHS system. Adding shortcuts in the main loop and including a single outer loop provided faster delivery speeds.

3 Finding the adequate number of vehicles to reach a stable wafer processing system was important. Several simulations were run at $125,150,200,250,300$, and 400 vehicles.

4 Providing additional vehicle capacity by using empty vehicle management algorithms.

5 Finding adequate start-up locations for the different equipment sets.

6 Finding an adequate amount of stockers.

\section{ANALYSIS OF 12-WAFER-LOT SYSTEMS}

\section{$5.1 \quad$ Experimental Design}

The 12-wafer-lot linked model was developed by using the 25 -wafer-lot linked model as the baseline (see Table 3). The resulting layout is shown in Figure 3. The levels of the AMHS factors constraining wafer processing were changed in order to increase AMHS capacity, considering the higher amount of moves expected in systems with smaller lot sizes.

More specifically, the 25 -wafer-lot baseline incurred the following modifications in order to create the 12-wafer-lot model:

- Due to the increased vehicle traffic around it, the outer loop was upgraded into a two-track traffic system in certain areas, especially near stockers.

- 12 additional stockers were added (for a total of 24 stockers), in response to higher WIP levels in a small- lot-size environment. Total stocker capacity was increased to 6590 lots (limited stocker capacity). 
- 18 stockers were positioned around the perimeter of the fab in order to minimize congestion at the inner loop.

- Connected the inner and outer loop to provide more alternate routes and direct transport connections.

- Empty vehicles in the main loop now circulate around the outer loop. As a result of these change, utilized vehicles can reach destinations between two different bays by using a less congested inner loop.

- The number of vehicles was increased to 250 vehicles, in response to a higher amount of moves requests received by the AMHS.

- Empty vehicles give higher priority to move requests, thus reducing the time that carriers wait to be picked up by an empty vehicle.

- The tool-to-tool settings were changed for a significant amount of tools, considering tools have different characteristics than those used in the corresponding 25-wafer-lot baseline.

- Tools were reassigned to stockers in order to rebalance the load at the stockers.

Table 3: Summary of experimental factors and factor levels used within the 12-wafer-lot linked model

\begin{tabular}{|c|l|c|c|}
\hline Code & \multicolumn{1}{|c|}{ Factor } & $\begin{array}{c}\text { 25-Wafer-Lot } \\
\text { Linked Model } \\
\text { Baseline }\end{array}$ & $\begin{array}{c}\text { 12-Wafer-Lot } \\
\text { Linked Model } \\
\text { Baseline }\end{array}$ \\
\hline A & \# of Vehicles & 125 & 250 \\
\hline B & \# of Stockers & 12 & 24 \\
\hline C & Stocker capacity (lots) & 2400 & 6590 \\
\hline D & Tool-to-tool move rate & $27 \%$ & $27 \%$ \\
\hline E & Inner loop & Single & Single \\
\hline F & Outer loop & Single & Single (2-Track) \\
\hline G & Empty vehicle control & ZB & ZB \\
\hline
\end{tabular}

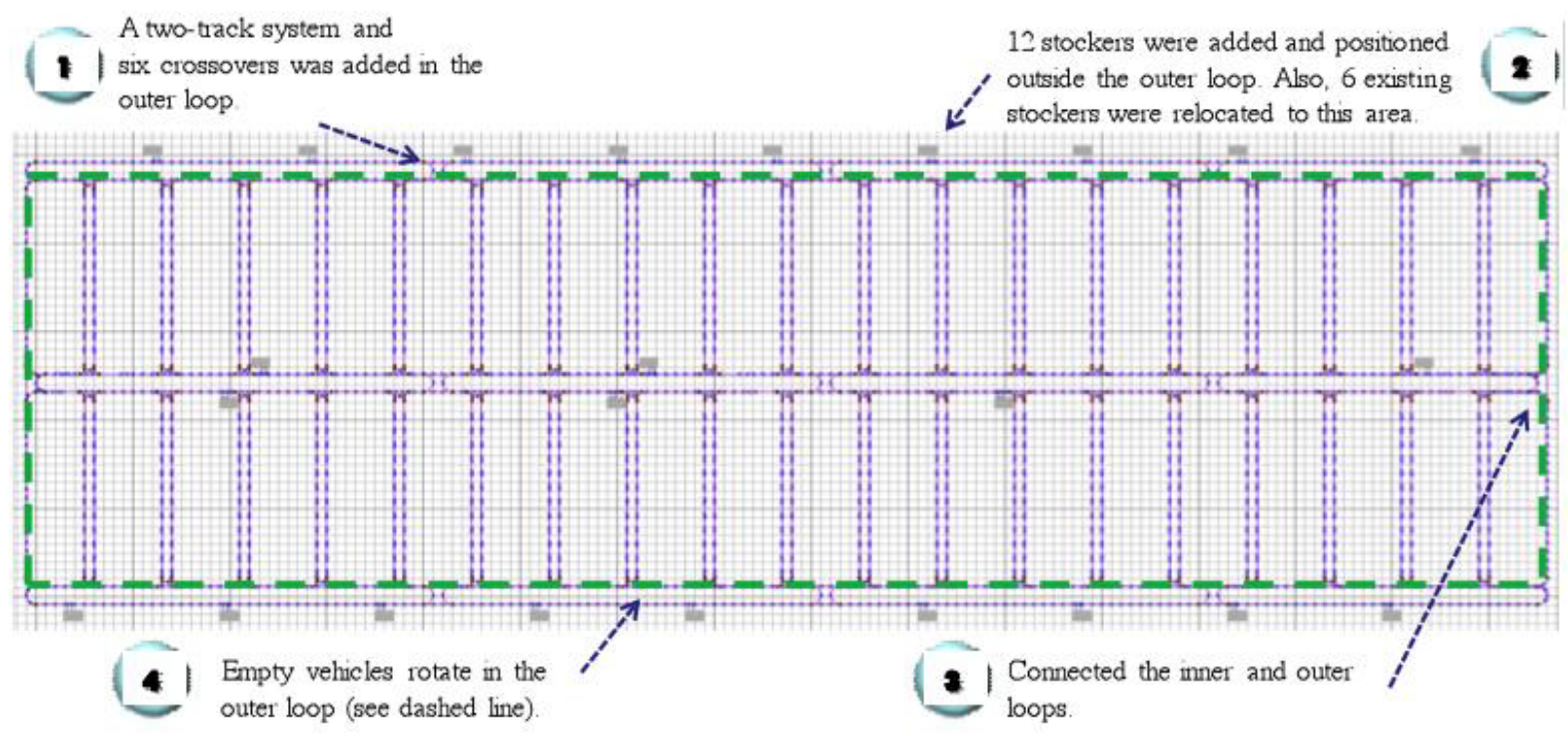

Figure 3: Spine layout configuration for 12-wafer lot system 
Jimenez, Wright, Grosser, Bell, Adikaram, and Davila

\subsection{Summary of Results}

The results of the 12-wafer-lot baseline are shown in Table 4 . For convenience of the reader, this table also replicates the results of the 25 -wafer-lot baseline, both at 125 and 150 vehicles. These results were obtained by running the simulation model for 750 simulation days, including a warm-up period of 250 days. Each independent model was stable.

Table 4: Summary of results for the 12-wafer-lot linked model

\begin{tabular}{|c|c|c|c|c|c|c|}
\hline Model & $\begin{array}{c}\text { Vehicle } \\
\text { Utilization } \\
\text { (\%) }\end{array}$ & $\begin{array}{c}\text { Average } \\
\text { Stocker } \\
\text { Load } \\
\text { (lots) }\end{array}$ & $\begin{array}{c}\text { Total } \\
\text { Stocker } \\
\text { Load } \\
\text { (lots) }\end{array}$ & $\begin{array}{c}\text { Moves } \\
\text { Per } \\
\text { Hour }\end{array}$ & $\begin{array}{c}\text { Retrieve } \\
\text { Time } \\
\text { (sec.) }\end{array}$ & $\begin{array}{c}\text { Transport } \\
\text { Time } \\
\text { (sec.) }\end{array}$ \\
\hline $\begin{array}{c}\text { 25-Wafer-Lot Baseline } \\
(125 \text { veh/12 stk/2400 stk cap) }\end{array}$ & 69 & 52 & 622 & 1717 & 136 & 83 \\
\hline $\begin{array}{c}\text { 25-Wafer-Lot Baseline } \\
(150 \text { veh/ 12 stk/ 2400 stk cap) }\end{array}$ & 56 & 53 & 636 & 1723 & 133 & 85 \\
\hline $\begin{array}{c}\text { 12-Wafer-Lot Baseline } \\
(250 \text { veh/24 stk/ 6590 stk cap })\end{array}$ & 47 & 41 & 972 & 2964 & 44 & 89 \\
\hline
\end{tabular}

From the AMHS standpoint, the changes made to the 12-wafer-model baseline improved the system performance considerably. The extra $2443.6-\mathrm{ft}$ of track that was added to the system, the reduction of areas with high traffic concentrations (e.g. near stockers) and the improvements in vehicle logistics resulted in a 5-second increase in transport time. Such increase is small considering that the number of moves per hour increased by 72 -percent and there are twice as many vehicles than in the 25 -wafer-lot baseline (125 vehicles). Similarly, system improvements lead to approximately $67 \%$ decrease in the retrieve times. The total number of carriers stored at stockers increased due to the higher WIP levels of the 12-wafer-lot system; however, the number of carriers per stocker dropped by almost $22.7 \%$ due to the additional stockers. In part, doubling the stocker count also contributed to lower retrieve times because there are fewer carriers per stocker load port. The vehicle utilization for the 12-wafer-lot AMHS is 47.2\%. Such decrease represents approximately $15-24 \%$ less utilization than what is observed in the 25 -wafer-lot baseline, providing flexibility to decrease the vehicle count.

\subsection{Lessons learned on 12-wafer-lot models}

The development of the 12-wafer-lot model had similar challenges to those of the 25-wafer-lot model. The top critical changes to the 12-wafer-linked models were:

1 Determining the tools to be configured with a tool-to-tool setting.

2 Doubling number of vehicles and stockers provided sufficient vehicle and storage capacity to account for the increased WIP levels.

3 Adding additional track, moving stockers to the outer loop, and improving the vehicle logistics provided faster delivery speeds.

\section{CONCLUSIONS}

This paper identifies the AMHS factors that limit the productivity of 12-wafer-lot systems. These factors comprise the track layout, number of vehicles, empty vehicle management rules, number of stockers, and stocker capacity. These factors were evaluated and configured using linked simulation models. This study reports the lessons learned gained from our simulation analysis. In fact, our studies with a 12 -wafer-lot virtual fab demonstrated that the AMHS is capable of supporting small lot operations if congestion is minimized around high traffic areas. The main solutions investigated included adding additional track around high-throughput stockers, increasing the amount of transports in the AMHS, and improving the control of empty transports. This work is part of a long-term research effort to design next generation 
Jimenez, Wright, Grosser, Bell, Adikaram, and Davila

semiconductor wafer fabs. In our future research, our simulation studies will incorporate and evaluate the performance of four-track systems and undertrack storages.

\section{ACKNOWLEDGEMENTS}

This work was funded by SEMATECH, Inc., under agreement \#: 100158-MM.

\section{REFERENCES}

Adusumilli, K. M., and R. Wright. 2004. Comparative factory analysis of standard FOUP capacities. In Proceedings of the 2004 Winter Simulation Conference, ed. R.G. Ingalls, M.D. Rossetti, J.S. Smith, and B.A. Peters, 1930-1934. Piscataway, New Jersey: Institute of Electrical and Electronics Engineers, Inc.

Bass, E., and R. Wright. 2008. Modeling semiconductor factories for equipment and cycle time reduction opportunities. Future Fab International 24:50-55.

Glüer, D. 2003. Optimization of automated material handling systems regarding robustness and delivery time. IEEE/SEMI Advanced Manufacturing Conference, 96-100.

Marshall, L., K. Rust, and K. Schmidt. 2007. 300mm prime gaps that need to be addressed to boost productivity. International Symposium on Semiconductor Manufacturing, 1-4.

Pettinato, J., and D. Pillai. 2005. Technology decisions to minimize 450-mm wafer size transition risk. IEEE Transactions on Semiconductor Manufacturing 18:509-501.

Pillai, D. 2006. The future of semiconductor manufacturing. IEEE Robotics and Automation Magazine 13:16-24.

Wright, D. R., and T. Chang. 2006. Cycle time perspectives for small transfer batch size. The 17th Annual SEMI/IEEE Advanced Semiconductor Manufacturing Conference, 312-315.

Zimmerhack1, O., J. Rothe, K. Schmidt, and L. Marshall. 2007. The effects of small lot manufacturing on AMHS operation and equipment front-end design. International Symposium on Semiconductor Manufacturing, 1-5.

\section{AUTHOR BIOGRAPHIES}

JESUS A. JIMENEZ is an Assistant Professor in the Industrial Engineering program of the Ingram School of Engineering at Texas State University-San Marcos. He obtained his Ph.D. in Industrial Engineering from Arizona State University, and his M.S. and B.S. in Industrial Engineering from The University of Texas at El Paso. He is director of the Center for High Performance Systems (CHiPS) at Texas State University-San Marcos, where he conducts research in factory logistics, automated material handling systems, and the modeling and analysis of manufacturing systems, especially semiconductor manufacturing. His email address is <jesus.jimenez@txstate.edu>.

ROBERT WRIGHT manages factory simulation projects for International SEMATECH Manufacturing Initiative (ISMI). He has 15 years experience in simulation and cost modeling and is a senior member of the technical staff. Mr. Wright received his BBA in management and his M.S. in industrial technology from Texas State University, San Marcos. Through ISMI, he has supported the CHiPS lab at Texas State University. His email address is <robert.wrighteismi.sematech.org>.

ALEXANDER GROSSER obtained his degree in Electrical Engineering from the University of Applied Science in Giessen-Friedberg Germany. He joined AMD/GLOBALFOUNDRIES Diffusion module in 2004 and started as a Manufacturing Engineer. Alexander was a member of the Fab36 StartUp team in Dresden Germany. In 2008, he joined the AMD Industrial Engineering group. His two year assignment at 
ISMI in Austin, TX was focused on Small lot sizes operation studies. His email address is <alexander.grossereglobalfoundries.com>.

MICHAEL A. BELL obtained his B.S. in Industrial Engineering from Texas State University-San Marcos. While earning his degree he held a research position at the CHiPS Lab. His email address is $<$ mb1425etxstate.edu>.

CHARITHA ADIKARAM is an undergraduate research assistant at the CHiPS Lab. He is pursuing his Bachelor of Science degree in Electrical Engineering with a concentration in Micro and Nano Devices and Systems at Texas State University-San Marcos (Class of 2013). His email is address is $<$ ca1276etxstate.edu>.

VICTORIA DAVILA is currently an operations engineer for Lockheed Martin. She obtained her B.S. in Industrial Engineering from Texas State University-San Marcos. While pursing her degree she maintained a position as simulation analyst for International SEMATECH Manufacturing Initiative and the CHiPS Lab. Her email address is <vd1023@gmail. com>. 